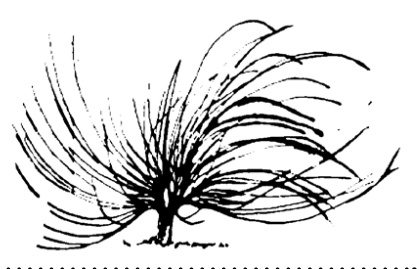

\title{
Aprendizaje con herramientas TIC: un nuevo desafío en tiempos de COVID-19, en estudiantes de la Universidad Nacional de Costa Rica, Sede Región Chorotega
}

\author{
Carlos Luis Chanto Espinoza ${ }^{l}$ \\ Universidad Nacional \\ Costa Rica \\ carlos.chanto.espinoza@una.cr \\ Jorge Luis Loáiciga Gutiérrez ${ }^{2}$ \\ Universidad Nacional \\ Costa Rica \\ jorge.loaiciga.gutierrez@una.ac.cr
}

\begin{abstract}
Resumen
En el ámbito de la investigación, el proceso de enseñanza en tiempos de pandemia ha tenido una transformación mundial, estableciéndose como eje esencial el apoyo de herramientas virtuales. El proceso de la enseñanza, apoyado de las Tecnologías de Información y la Comunicación (TIC) $)^{3}$, ha traspasado en todos los contornos y en múltiples horizontes educativos, desde el aprendizaje
\end{abstract}

Recibido: 5 de octubre de 2020. Aprobado: 1 de junio de 2021

http://dx.doi.org/10.15359/rep.16-2.8

1 Académico, investigador de la Universidad Nacional. Doctor en Proyectos. Máster en Gerencia y Administración de Proyectos de Desarrollo. Maestría en Dirección Estratégica. Especialidad en Tecnologías de la Información, máster en Dirección Estratégica en Tecnologías de la Información. Licenciado en Informática con énfasis en Sistemas de Información. http://orcid. org/0000-0002-3420-7259

2 Académico, investigador de la Universidad Nacional. Doctorando en Proyectos con Especialidad en Investigación por la UNINI, máster en Administración de Proyectos por la UCI. Licenciado en Administración con énfasis en Gestión Financiera por la UNA. Certificación Internacional Green Project Management por el GPM-Global. https://orcid.org/0000-0002-0421-3906

3 Léase de ahora en adelante TIC como: Tecnologías de la Información y la Comunicación. 
informal hasta el formal. El objetivo de este artículo fue conocer el aprendizaje con herramientas TIC y el nivel de percepción en torno a la realización de cursos en modalidad virtual frente a la pandemia del COVID-19. Para lograrlo se realizó un estudio cuantitativo con una muestra de 115 estudiantes de la Universidad Nacional de Costa Rica (UNA), Sede Región Chorotega, mediante una técnica de muestreo, no probabilística, por conveniencia con un estudio descriptivo y explicativo. Las conclusiones de este análisis muestran un nivel aceptable en el aprendizaje con herramientas TIC y una adaptabilidad a la virtualidad, en cuanto al estudio del aprendizaje de los educandos en balance con el modelo tradicional o de enseñanza presencial. Además, no resulta sencilla su integración en un entorno virtual colaborativo.

Palabras clave: Aprendizaje activo, educación, enseñanza, innovación pedagógica, TIC

\begin{abstract}
In the field of educational research, the teaching process in times a pandemic has undergone a global transformation, establishing the support of virtual tools as an essential axis. The process of teaching supported by Information and Communication Technologies (ICT) has crossed in all contours and in multiple educational horizons, from informal to formal learning. The objective of this article was to know the learning with ICT tools and the level of perception around the realization of courses in virtual modality, during the COVID-19 pandemic. To achieve this, a quantitative study was carried out with a sample of 115 students from the National University of Costa Rica (UNA), Chorotega Branch Campus, using a sampling technique, not probabilistic, for convenience with a descriptive and explanatory study. The conclusions of this study show an acceptable level in learning with ICT tools and an adaptability to virtuality in terms of the study of student learning in balance with the traditional or face-to-face teaching model. Furthermore, its integration into a collaborative virtual environment is not easy.

Keywords: education, experiential learning, ICT, teaching method innovations, teaching process
\end{abstract}




\section{Introducción}

$\mathrm{L}$

a pandemia del Covid-19 ha establecido un gran desafío para las instituciones de educación superior. A partir de la detección del primer caso de Covid-19 en Costa Rica los campus universitarios cerraron sus puertas y el proceso de enseñanza-aprendizaje se transformó. Las instituciones de enseñanza tuvieron que adoptar nuevas lógicas y dinámicas de formación, al transformar la transferencia del conocimiento de una modalidad presencial a una modalidad virtual.

Hoy, en tiempos de incertidumbre y aislamiento social por efectos de Covid-19, en el marco de la pandemia, la situación actual, en el orbe, presume un desafío hasta hace muy poco inconcebible para muchos escenarios educativos. Los cuales se vieron forzados a cerrar sus puertas buscando mitigar el avance del Covid-19. Dentro de esta nueva normalidad, cada docente y educando debe adecuarse a la enseñanza en dimensiones no presenciales.

En el contexto mundial, los conflictos que encierra este reto se ven incrementados por las discrepancias socioeconómicas, con las invariables brechas digitales, entre otras, tanto para educandos como el profesorado.

Las destrezas de aprendizaje poseen un papel concluyente para el alcance de la independencia, la autonomía epistemológica y así, adjudicarse el modelo activo del proceso de enseñanza-aprendizaje en que están sumergidos. La ejecución de entornos virtuales es una estrategia innovadora para obtener, en los educandos, el aprendizaje. Los entornos virtuales de educación general y comunicación pedagógica componen un marco en el cual se originan habilidades de aprendizajes, como elementos que establecen la aceleración y ordenación del aprendizaje desarrollador:

Hoy en día la Educación Superior fomenta la incorporación de las tecnologías educativas en sus procesos de enseñanza-aprendizaje y en sus prácticas tanto dentro como fuera del aula. La formación del docente universitario se constituye en una de las áreas estratégicas de las universidades para el mejoramiento de la calidad educativa, y el uso de las TIC se convierte en una alternativa para el desarrollo de competencias del profesorado tanto para la investigación, la docencia, la gestión académica y la vinculación con la sociedad. (Balladares-Burgos, 2018, p. 42) 
En este sentido, "el profesorado de hoy debe ser capaz de utilizar las tecnologías y asimilar la importancia que tiene el fenómeno de las redes sociales digitales en nuestro presente y nuestro futuro" (Iglesias et al., 2013, p. 335).

Asimismo, Monasterio y Briceño (2020) formulan que "[a]nte la situación de la pandemia, las organizaciones educativas a nivel mundial encuentran una alternativa en la educación mediada por las tecnologías, sin embargo, en algunas de estas instituciones esta modalidad se encuentra incipiente y poco desarrolladas" (p. 104).

La pandemia de COVID-19 obligó a los centros de educación superior a tomar medidas de aislamiento, lo cual impactó en su totalidad a educandos en todo el mundo. Por ende, los esfuerzos generados por las autoridades de salud, para efectos de detener la propagación de COVID-19, a través del autoaislamiento y distanciamiento social, han inducido a la utilización de metodologías de innovación, con ayuda de herramientas TIC.

Producto del aislamiento social, la Universidad Nacional ha asumido el desafío de la educación virtual, tomando medidas para adoptar un enfoque online que satisfaga las necesidades de educación. En este marco podemos mencionar algunas investigaciones de diversos autores que hacen referencia a la utilización de las TIC antes y en tiempos de COVID-19.

\section{Marco teórico}

Los contextos del nuevo mundo, donde el conocimiento es el activo más significativo de la humanidad, causan que el sumario de enseñanza, así como las instituciones de educación ejerzan un compromiso general de innovar en su quehacer pedagógico y sus formas de gestionar el aprendizaje. Estos componentes exigen la figura de docentes con experiencias interdisciplinarias, con modernos e imperecederos enfoques metodológicos y con más capacidad para la utilización racional y conveniente de las herramientas disponibles, fundamentalmente de las TIC.

Desde los postrimeros años, se han consolidado modelos de enseñanza con herramientas TIC, así como de alineación continua que inclusive en tiempos atrás eran posibles en un nivel pequeña y con costos exageradamente elevados. 
Para lograr un eficiente y eficaz proceso enseñanza aprendizaje a través de las TIC no basta con el uso y aplicación de la tecnología, es necesario desarrollar modelos educativos que combinen medios, métodos y formas de enseñanza, que aprovechen las ventajas para la colaboración humana. La incorporación de la didáctica en la enseñanza, el uso de pedagógico de las TIC y la aplicación de las teorías constructivistas han permitido que algunos profesores modifiquen sus aulas al introducir estas metodologías que pueden tener diversos nombres, ya sea, "aprendizaje activo" propiamente dicho, "aula invertida", "aprendizaje a partir de problemas", entre otras (Moreno-Correa, 2020, p. 17).

De esta forma, Ramírez y Barragán (2018) manifiestan: "El avance de las tecnologías digitales en los últimos años y su empleo progresivo en el ámbito educativo han permitido el desarrollo de estrategias didáctico-pedagógicas para su inclusión en la educación formal desde el nivel básico hasta el posgrado" (p. 95).

En todo caso, López-Neira (2017) advierte que la utilización de las TIC como herramientas de apoyo en la docencia, deberían ser a partir de un modelo constructivista:

En la actualidad existen diversas herramientas digitales o ambientes interactivos de funcionamiento en Internet, que permiten implementar actividades de aprendizaje bajo un modelo constructivista, en las cuales los estudiantes pueden construir su propio conocimiento a través de la elaboración de productos digitales (videos, animaciones, modelos, etc.) o la generación de estrategias para resolver desafíos o problemas a través de ambientes lúdicos. (p. 98)

Con la incorporación de las TIC se han creado nuevos contextos que ofrecen la facilidad de edificar ambientes de enseñanza y aprendizaje que brinden, lo que denominamos, comunicación sincrónica, que es, en pocas palabras, el poder comunicarnos de manera simultánea. Esencialmente, dichas posibilidades comunicativas admiten importar metodologías de colaboración virtual en una sociedad distinguida, por la interconectividad, hipertextualidad, y multiculturalidad del nuevo siglo.

Con base en lo anterior, se menciona: 
La educación, tal y como hemos expuesto anteriormente, se ha visto sometida a diversos paradigmas que han revolucionado tanto la forma de entender dicho fenómeno como su finalidad. Actualmente, el nuevo reto al cual se enfrenta la educación reside en su convivencia con el creciente desarrollo de las TIC y su coexistencia con los "recursos tradicionales". (Blancafort et al., 2019 p. 53)

En la misma línea, "en contraste con la educación tradicional, las opciones pedagógicas y didácticas con apoyo en las TIC tienen mayor impacto, haciendo más eficiente lo que tradicionalmente se ha venido haciendo" (Hermosa, 2015, p. 123).

En el caso específico y con el objetivo de apoyar el proceso de enseñanza - aprendizaje de la Universidad Nacional Sede Región Chorotega, se han puesto a disposición de la comunidad universitaria algunas herramientas tecnológicas:

Office 365: Plataforma de comunicación, productividad, y colaboración. Pack de ofimática online de la empresa Microsoft, que presta servicios en la nube de las diferentes aplicaciones (Excel, Word y PowerPoint y OneNote).

Zoom (Video Conferencing, Web Conferencing, Webinars): Es una herramienta para realizar conferencias, contiene múltiples funcionalidades (audio, chat, vídeo compartir contenido, adicional a otras formas de interactuar con los partícipes). Permite hacer grabaciones de las sesiones, compartirlas. Y, finalmente, admite crear salas virtuales para trabajo en equipos.

Microsoft Teams: Microsoft Teams es una plataforma en la que se agrupan múltiples aplicaciones tales como llamadas, calendario, reuniones virtuales, chat, almacenamiento de archivos, trabajo colaborativo de documentos en tiempo real, integración de aplicaciones de Microsoft y de otras organizaciones.

Google Hangouts Meet: Plataforma de videoconferencias de la empresa Google. Orientada para la utilización en navegadores web, así como dispositivos móviles. Basada al contexto educacional, como empresarial y viene dentro del paquete de G-Suite.

Aula Virtual: Espacio virtual online, mediante la cual los docentes-educandos tienen a disposición múltiples herramientas 
tecnológicas, que colaboran en el desarrollo de los procesos de enseñanza y aprendizaje.

Las TIC aplicadas a la formación pedagógica como herramienta de virtualización, en términos generales, busca conseguir los objetivos planteados, tales como: optimizar la enseñanza y, por consiguiente, obtener un alto y estable beneficio académico del educando; esto tiene en cuenta que, como toda herramienta, se debe estar al corriente de su implementación o podría brindar factores inversos a los planteados.

Granda et al. (2019) indican,

Estas tecnologías en el contexto educacional desempeñan un rol fundamental y son cada vez más imprescindibles en el acceso universal al conocimiento, han sido factor determinante en la democratización de la enseñanza; su adecuado empleo contribuye a brindar un aprendizaje de calidad. (p. 105)

Tal y como lo mencionan Villacres et al. (2020): "Sin lugar a dudas, la presencia, cada vez más común de las TIC en el campo de la educación y por otro lado, la gran velocidad de su desarrollo y la novedad que representan despiertan miedos y resistencias de algunos discentes" (p. 137).

Por consecuencia, como señalan Espinoza et al. (2019) y Espinoza et al. (2020), el proceso de enseñanza-aprendizaje no ha sido ajeno a esta metamorfosis, y en los últimos años han nacido variadas experiencias concernientes a la integración de las TIC en la educación.

En su defecto Márquez y Mancilla (2016): "es imperativo procurar el desarrollo de nuevos procesos mentales que permitan, a las actuales generaciones, crear paradigmas para respaldar el surgimiento y aplicación de innovadoras tecnologías" (p. 30).

El mayor desafío que presenta la utilización de las TIC como herramientas de apoyo tecnológico en el salón de clases consiste en "ajustar" las mejores destrezas de la formación tradicional y adaptarlas a la realidad de tiempo y espacio. Este proceso lo podemos denominar como "reinvención", con el objetivo de propagar efectivamente la mejor característica de cada tipo de enseñanza. En este amplio marco, la educación tradicional presenta un carácter riguroso e inflexible, en donde la virtualidad muestra a los actores una serie de contextos más 
extensos y dúctiles que los exigen no solo a recoger contenido, sino ser parte de un proceso más práctico y riguroso.

En este contexto, es imprescindible la incorporaración de las TIC a la educación superior, debido a que en pleno siglo XXI forman parte de nuestra vida cotidiana:

La educación con TIC no se limita al uso de una herramienta, sino que implica un conjunto de competencias de gestión de información, de comunicación y de intercambio. Por ello es necesario elaborar propuestas didácticas que preparen a los alumnos en el uso comprensivo y crítico de la tecnología que los rodea. (Verdecia et al., 2015, p. 13)

Por ende, la experiencia educativa virtual se alimenta de métodos de aprendizaje achacables a la práctica presencial como unidad para crear aprendizaje. No obstante, la virtualidad ejerce un sumario de construcción firme de retención de conocimientos, en donde el entorno virtual tiene su valor, así como la participación de otros cómplices, entre ellos, la interpretación de la información.

Los métodos educativos, de la misma forma que otras técnicas humanas, tienen una irrefutable predisposición a desarrollarse hacia horizontes inapreciables y de acuerdo con las permutas de la dinámica humana. Es importante definir que un desconocido componente se adiciona a estos métodos educativos e incumbe a un factor oportuno de la modernidad; el cual es denominado como flexibilidad.

De esta manera, es necesario conocer una experiencia de aprendizaje que incorpore ambientes digitales a consecuencia del COVID-19:

Es evidente que la implementación de las Tecnologías de Información y Comunicación (TIC), se constituye en un factor de expansión, de desarrollo y evolución de las nuevas formas de enseñanza y aprendizaje de hoy y del futuro, en las cuales el docente es una pieza fundamental y articuladora de su uso, fundamentado en sus competencias, saberes y la disciplina que de por sí domina. (Cardona-Londoño et al., 2020, p. 45)

Las TIC como herramientas en el proceso de enseñanza-aprendizaje no pueden tomarse como una simple pericia educativa, que 
escuetamente incorpora un recurso o, en su defecto, un medio. El realizar labores pedagógicas con las TIC demanda un conjunto de condiciones económicas, didácticas, culturales, y políticas, para lograr que las mismas no sean consideradas simplemente como un marco informacional y comunicacional; no obstante, asimismo un espacio social.

Las TIC, por su biósfera, se han envuelto en múltiples actividades de la sociedad, lo que fomenta el desarrollo, genera nuevas prácticas de aprender y enseñar, así como las actividades sociales y laborales. Sin embargo, los disímiles sujetos del órgano educativo tenemos que modificar, de forma firme, el rol que ejercemos en el sistema, esto debido a que nuestras metodologías y destrezas deben desarrollarse en conjunto con el mundo y de la misma forma, establecer factores y objetivos de nuevos cambios e innovaciones.

Existe un pensamiento en que la educación persistentemente ocupa una mirada prospectiva, en otras palabras, que no solo tiene en cuenta el presente, sino que muestra una dimensión de futuro no definido de forma absoluta. No obstante, ese futuro para el cual nos preparábamos se cristalizó en nuestro presente de forma inédita y, abrupta, convirtiendo los espacios de formación docente en entornos virtuales, transformando la vida educacional, laboral y cotidiana.

El día a día se convirtió en renovadas destrezas personales y diferentes posibilidades de acceso y utilización de las TIC, donde súbitamente los recursos tecnológicos para la docencia se constituyeron en los salones de clases. Pero esto es todo un reto, ya que para cada uno de estos espacios virtuales se hace necesario tener conectividad a internet, dispositivos digitales, y muy importante el conocimiento de las herramientas tecnológicas, para lograr que circulen los saberes disciplinares y, al mismo tiempo, consigamos atender la dimensión pedagógica del educando.

\section{Metodología}

En la presente investigación, se utilizó un estudio orientado en un enfoque cuantitativo de fase descriptiva y explicativa. De esta forma el análisis "se fundamenta en un enfoque cuantitativo en los aspectos observables y susceptibles de cuantificar; es decir, que pueden ser contados. Se sirve de la estadística para el análisis de los datos" (Barrantes, 2015, p. 55). 
Según la profundidad o fase de la investigación, Barrantes (2015) menciona, "la investigación descriptiva tiene el objeto de describir los fenómenos y la fase explicativa trata de explicar los fenómenos y estudiar sus relaciones para conocer sus estructuras y los aspectos que intervienen en su dinámica" (p. 55).

Para cumplir con el estudio se estableció un cuestionario con preguntas cerradas y abiertas que permitieron dar validez al contenido de la investigación, a través de la plataforma tecnológica Google Forms, compartido por correo electrónico institucional y número telefónico, por medio de la aplicación de WhatsApp, a los estudiantes de la Universidad Nacional, Sede Región Chorotega, Campus Liberia, con el fin de conocer el aprendizaje con herramientas TIC y el nivel de percepción en torno a la realización de cursos en modalidad virtual frente a la pandemia del COVID-19. Los cuestionarios fueron aplicados en el período comprendido entre el 01 al 10 de agosto del 2020.

Por su parte, la población considerada es finita y compuesta por 320 estudiantes universitarios, donde la muestra seleccionada fue de 115 estudiantes del I al IV nivel de la carrera de Administración de la UNA, Campus Liberia. Se utilizó una técnica de muestreo no probabilística y por conveniencia, al usar como marco muestral el estudiantado matriculado y activo del I Ciclo del año 2020.

A continuación, en la Tabla 1 se muestra un resumen del proceso de recolección de información:

\section{Tabla 1}

Proceso de recolección de la información

\begin{tabular}{rccc}
\hline Método & Fase & Enfoque & $\begin{array}{c}\text { Téenicas y procedimiento } \\
\text { de recolección }\end{array}$ \\
Estadístico & $\begin{array}{l}\text { Descriptiva y } \\
\text { Explicativa }\end{array}$ & Cuantitativo & Encuesta (cuestionario) \\
\hline
\end{tabular}

Nota: Elaboración propia, (2020)

\section{Resultados, análisis y discusión}

El la Figura 1, por medio de los datos estadísticos descriptivos de 115 estudiantes que respondieron las preguntas se muestra que la mayoría de los estudiantes encuestados realizan sus actividades académicas en modalidad virtual desde el internet del lugar de habitación (71.3\% ); sin 
embargo, un $41.7 \%$ lo hace conectándose desde los datos de un celular pospago, además el $13.9 \%$ con internet de datos de celulares prepago, lo cual muestra condiciones de brecha digital y poco alentadoras respecto a la realidad que viven los estudiantes.

\section{Figura 1}

Fuentes de conectividad en los estudiantes encuestados de la UNA-SRCH

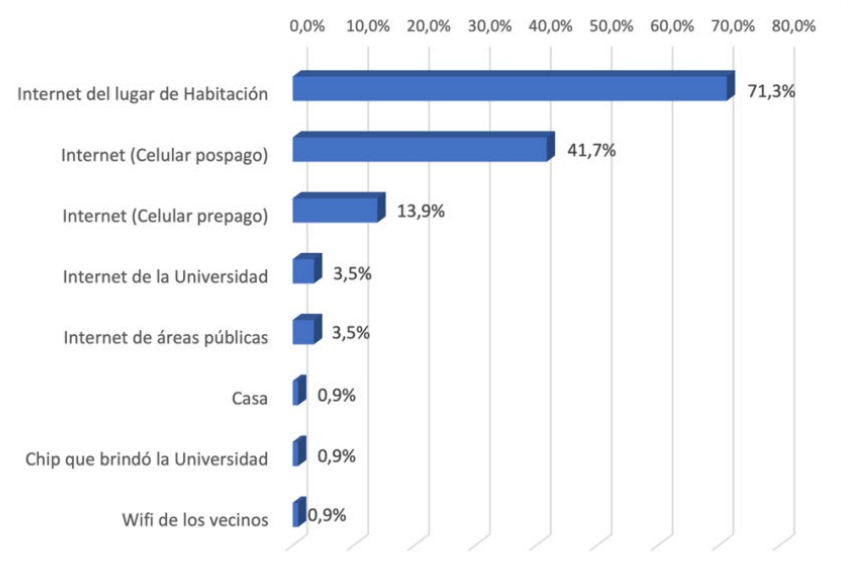

Nota: Elaboración propia, 2020

Un análisis relacional entre variables del cuestionario aplicado permite vislumbrar que los datos arrojados en la Figura 1 tienen origen en un total de 115 personas encuestadas de las cuales el $54.8 \%$ son del género femenino y el $45.2 \%$ del masculino, que su rango de edad oscila en un promedio de 17-22 años y que la mayoría reside en los cantones de Liberia (35.7 \%) y La Cruz (37.4\%), Guanacaste.

El dato más significativo es que el $53.9 \%$ de los estudiantes manifestó que esta era su primera experiencia recibiendo clases desde esta modalidad virtual, destinando un promedio de 4 a 6 horas diarias a las actividades asincrónicas (fuera de lo sincrónico, es decir, en tiempo real) desde la plataforma Aula Virtual de Moodle de la Universidad Nacional de Costa Rica (UNA). 
Por otra parte, la apropiación de la UNA de contar con Aula Virtual es muy favorable, para los estudiantes; en la Figura 2 se observa que, a la fecha, la plataforma de mediación pedagógica de mayor uso es el Aula Virtual Institucional (91.3\%), seguido de plataformas como Zoom (90.4 \%) y de Google Hangouts Meet (80.9 \%).

\section{Figura 2}

Implementación de plataformas de mediación pedagógicas de la UNA-SRCH

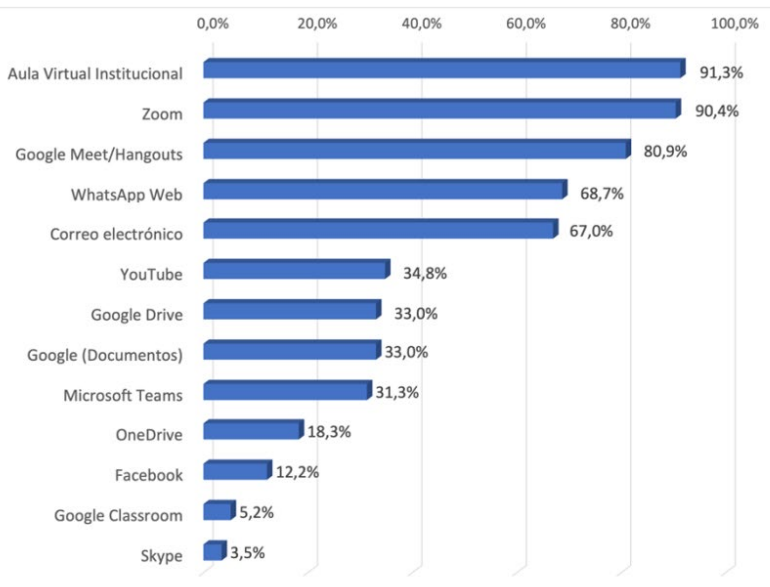

Nota: Elaboración propia, 2020

En este orden de ideas, el $88.7 \%$ de los estudiantes encuestados consideran que de las mejores estrategias implementadas por los docentes en las clases son las teleconferencias o videollamadas en tiempo real o sincrónico, combinado de actividades asincrónicas como los foros en línea, tareas en grupo, lecturas en la web, libros electrónicos y demás recursos y actividades que facilitan el entorno virtual colaborativo del Aula Virtual de la UNA, frente al $39.1 \%$ de videos pregrabados por los docentes, videos en la web y documentos escaneados. Son datos que permiten situaciones de mejora en la academia.

Por tal motivo, el ejercicio docente debe ser repensado, generando nuevas metodologías de enseñanza y procesos didácticos 
adaptados al entorno de la sociedad actual y a las necesidades de la particularidad de sus alumnos, que garanticen una educación inclusiva y de calidad; a través del uso de herramientas tecnológicas que permitan generar nuevas alternativas que orienten, ejecuten y evalúen los procesos de enseñanza y de aprendizaje. (Calderón et al., 2018, p. 37)

En relación con el grado de motivación de los estudiantes en el proceso de aprendizaje desde una modalidad virtual, en general manifestaron que les ha sido fácil adaptarse a las clases en línea, pero que el proceso tiene etapas de aprendizaje en donde han tenido que dedicar más tiempo de estudio a los cursos, estableciendo suficiente atención por el profesorado.

Los estudiantes sugieren que la Universidad Nacional Sede Regional Chorotega debe mejorar en el proceso de brindar clases en una modalidad remota por lo que en la Figura 3 se sugieren medidas concretas.

\section{Figura 3}

Sugerencias de medidas concretas a la UNA-SRCH

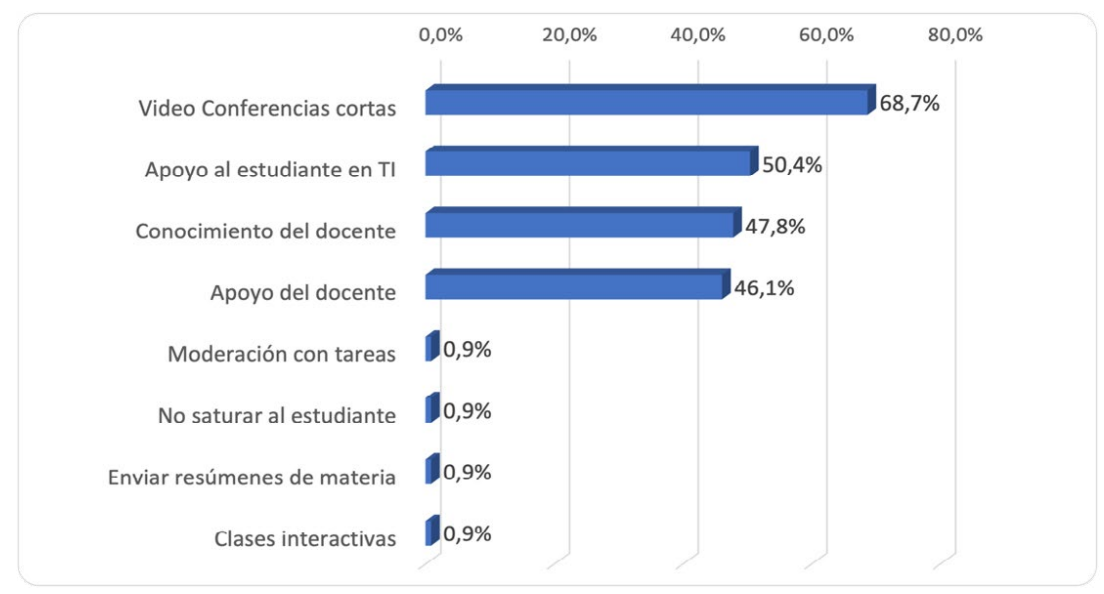

Nota: Elaboración propia, 2020 
A continuación, se enumeran, las medidas más representativas:

- $\quad$ El $68.7 \%$ sugiere mantener las videoconferencias cortas durante las clases.

- $\quad$ Un $50.4 \%$ solicita el apoyo a la persona estudiante, en el conocimiento de la tecnología a utilizar.

- $\quad$ El $47.8 \%$ considera importante mejorar el conocimiento de la persona docente en el uso de la tecnología a utilizar.

- $\quad$ El $46.1 \%$ requiere mayor apoyo de la persona docente.

- $\quad$ Al considerar estas medidas, es importante destacar que "El uso de TIC por parte de los docentes, se ve menguado en la medida en que ellos, según la percepción de los estudiantes, dan mayor relevancia al trabajo orientado presencialmente" (Ñáñez-Rodríguez et al., 2019, p. 107).

En otra de las preguntas realizadas, se solicitó a los estudiantes mencionar dos herramientas tecnológicas que se les dificultara utilizar y, en su mayoría, presentan debilidades en la hoja de cálculo de Excel y Microsoft Teams; además, se emitieron criterios importantes a evaluar como por ejemplo: el participante número 85 afirmó "A mí no, pero me gustaría que los profesores dominaran más el aula virtual y teams, ya que algunos aún nos hacen usar apps externas, y no es cómodo en 1 mismo día conectarse a 3 apps distintas, enviar tareas por correo, aula virtual y whatsapp".

En general, un $68.7 \%$ del estudiantado indica entender lo justo y necesario en cuanto al nivel de conocimiento de las herramientas tecnológicas. Sin embargo, siempre hay carencias sobre herramientas digitales: En la Tabla 2, se muestra un extracto de las diferentes percepciones sobre habilidades y carencias encontradas. 


\section{Tabla 2}

Extractos de las percepciones del estudiantado de sus habilidades y carencias sobre las herramientas digitales

\begin{tabular}{|c|}
\hline $\begin{array}{l}\text { Me cuesta un poco, pero con } \\
\text { práctica logro entender a la } \\
\text { perfección. }\end{array}$ \\
\hline $\begin{array}{l}\text { Tengo la habilidad que me adapto } \\
\text { muy fácil a la tecnología y } \\
\text { aprendo rápido. }\end{array}$ \\
\hline $\begin{array}{l}\text { Me desenvuelvo bastante bien } \\
\text { en el manejo de herramientas } \\
\text { digitales, ya sean ofimáticas, de } \\
\text { investigación, educativas, y de } \\
\text { edición, hay cosas que aún no } \\
\text { logro comprender, pero se trata de } \\
\text { aprender y adaptarse. }\end{array}$ \\
\hline $\begin{array}{l}\text { Como habilidad tengo la } \\
\text { navegación en internet. Carencia, } \\
\text { me cuesta instalar programas. }\end{array}$ \\
\hline $\begin{array}{l}\text { Habilidades, creo que domino las } \\
\text { herramientas muy bien, carencias } \\
\text { creo que no tengo. }\end{array}$ \\
\hline Ninguna por el momento. \\
\hline $\begin{array}{l}\text { Manejo bien internet y los } \\
\text { programas de Word, Excel, } \\
\text { PowerPoint y TIC. }\end{array}$ \\
\hline
\end{tabular}

No tengo habilidades, ni sé porque lo sigo intentando.

Admito que he mejorado en realizar trabajos escritos y en realizar presentaciones creativas, sé utilizar todas las herramientas correctamente, mi único problema es que tengo un internet un poco malo, porque a veces se me va y mi computadora a veces se me apaga, pero nada más.

Las herramientas dan los pasos a seguir, suele ser sencillo de interpretar, pero aun así no tengo conocimiento total en asuntos digitales o software.

Sé instalar software y sé algo de Excel, pero muy poca para mi gusto.

Habilidades es que aprendo rápido a utilizarlas y como carencias es que tengo que andar pidiendo prestados celulares porque el mío no me permite bajar aplicaciones.

Tengo ciertos conocimientos. Me cuesta mucho el manejo de tecnología ya que crecí $\sin$ nada de eso y no pude aprender tampoco por la falta de recursos tecnológicos. 


\begin{tabular}{|l|}
\hline Mala conexión, aprendo rápido. \\
\hline $\begin{array}{l}\text { Falta de conocimiento de las } \\
\text { herramientas y funciones que } \\
\text { poseen, facilidad y disposición de } \\
\text { aprender. }\end{array}$ \\
\hline $\begin{array}{l}\text { Solo sé lo básico y el internet muy } \\
\text { poco ayuda. }\end{array}$ \\
\hline $\begin{array}{l}\text { No tengo una computadora que } \\
\text { funcione, y aunque tengo el } \\
\text { celular se me dificulta. }\end{array}$ \\
\hline $\begin{array}{l}\text { Habilidades básicas en el uso } \\
\text { básico de herramientas de } \\
\text { cualquier programa o aplicación } \\
\text { que uso. Carencias en conocer } \\
\text { más herramientas de trabajo } \\
\text { para ampliar el conocimiento } \\
\text { de mejoras en el manejo de los } \\
\text { recursos. }\end{array}$ \\
Na: Elabocion propia (2020), pan
\end{tabular}

Me cuesta mucho el manejo de tecnología ya que crecí sin nada de eso y no pude aprender tampoco por la falta de recursos tecnológicos.

La verdad no tengo mucha relación con la tecnología, pero las veces que me ha correspondido utilizarla, me he sabido defender y me adapto y aprendo rápido.

Me esfuerzo por aprender cada día más ante la situación que se está viviendo y la carencia es tal vez la falta de equipo tecnológico actualizado.

Habilidades: creatividad, pero todo muy básico. Carencias: conexión a internet regular, nunca he llevado un curso sobre computación.

Al menos yo cuento con la computadora, pero la verdad casi no sé usarla nada.

Nota: Elaboración propia (2020), a partir de la encuesta aprendizaje con herramientas TIC COVID-19

Como resultado del diagnóstico de las percepciones indicadas por los estudiantes encuestados se pueden generar tres variables importantes de considerar en cuanto a las experiencias en el aprendizaje con herramientas TIC en una modalidad virtual en tiempos del COVID-19: 


\section{Figura 4}

Sistematización de las percepciones por el estudiantado encuestado

\section{La brecha digital}
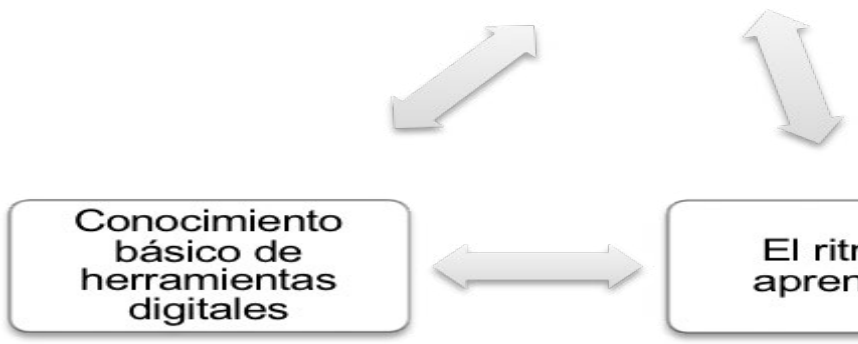

\section{El ritmo de aprendizaje}

Nota: Elaboración propia, 2020

El COVID-19 ha planteado un interesante reto para los estudiantes universitarios de la Universidad Nacional, Sede Región Chorotega, Campus Liberia, quienes no contaban con la experiencia de recibir clases en la presencialidad remota. A pesar de estas convergencias hay que ser conscientes que el uso de las herramientas digitales es vital para la continuidad de la educación universitaria y estas tres categorías principales (La brecha digital, el ritmo de aprendizaje, conocimiento básico de herramientas digitales) se consideran los principales retos identificados.

\section{Conclusiones}

Los hallazgos efectuados a través de la investigación y análisis de la literatura especializada, así como los resultados de la aplicación del cuestionario a los educandos nos permiten verificar que la implementación de las TIC, como herramientas apoyo en el proceso tanto de la enseñanza como del aprendizaje, es un hecho irrevocable, estableciéndose como un reto para los docentes y estudiantes.

La utilización de estas herramientas tecnologías como estrategia en el proceso de enseñanza y aprendizaje, en el marco de la muestra seleccionada de la Universidad Nacional Sede Regional Chorotega, Campus Liberia, se caracteriza por establecer la utilización de las TIC 
por parte de los educandos, como herramientas de apoyo para el desarrollo de sus lecciones; se centran, esencialmente, en computadoras y aplicaciones para video conferencias, presentaciones de diapositivas digitales y otros.

Es concluyente que en tiempos de pandemia el desafío no es tecnológico, ya que lo primordial se trata de una permuta única del proceso enseñanza y aprendizaje, en una sociedad de cambiantes factores de espacio y tiempo, que están variando el contexto de vivencia humana.

Sin duda alguna, a causa de las medidas de confinamiento y distanciamiento social, ante la contingencia COVID-19, las TIC evidenciaron su relevancia, y para muchos, tanto docentes como educandos, se han visto forzados a utilizarlas a marchas obligadas para la confección de materiales, utilización de sitios web y herramientas tecnológicas empleadas en el proceso de enseñanza aprendizaje.

Según los resultados, se ratifica que la principal herramienta TIC utilizada por parte de los estudiantes en su proceso de aprendizaje es la plataforma Moodle o Aula Virtual, (donde el docente les comparte recursos en relación con el curso), además clases en videoconferencia, a través de herramientas como Zoom, Teams, Google Hangouts Meet y WhatsApp, como una forma de comunicación más fluida.

Hoy día, el impacto de las TIC afecta a todos los contornos de nuestra vida. Existimos en una época de cambios que ocurren aceleradamente. Las tecnologías crean grandes variaciones e innovaciones que se plasman, de manera inevitable, en nuestro ambiente. Las TIC personificadas por espacios de multimedia interactivas con su alianza a las redes componen un eje significativo en la transformación subyacente que está cambiando el mundo actual. La combinación de imagen, textos, y sonido, brindan las posibilidades de colaboración activa del educando. El marco multimedia interactivo que proponen las TIC muestra un gran potencial para el desarrollo de recursos y aplicaciones pedagógicos.

Los resultados indican que el proceso educativo no puede detenerse. La Universidad Nacional tiene que extender los recursos para innovar en el sumario de enseñanza-aprendizaje y garantizar la marcha en los planes de estudio, en donde los docentes deben alfabetizarse en el manejo de las TIC. De la misma forma, los diseños de plataformas virtuales deben asumir un reto pedagógico interdisciplinar. No debe existir restricción en la implementación de diferentes plataformas para apoyar al educando en su proceso formativo. 
Se establece que el aprendizaje colaborativo y la disponibilidad de información son elementos esenciales, que los educandos mencionan las TIC como herramientas para establecer la comunicación entre los múltiples sujetos en el proceso de enseñanza aprendizaje. De las desventajas que se establecen, se encuentran las fallas en la conexión de internet y las múltiples distracciones durante la clase en sus hogares, lo que ocasiona un bajo rendimiento en su aprendizaje.

Las reglas gubernamentales encaminadas hacia la prevención de la infección por COVD-19 han impactado fuertemente en los múltiples programas académicos, al suspender procesos académicos y requerir el éxodo hacia metodologías de aprendizaje por medios virtuales, al fundamentarse que no se tiene una idea de fecha del retorno para restablecer la presencialidad.

El desafío es arduo, pero se establece como una práctica enriquecedora que alcance a derribar los mitos y barreras que muchos estudiantes han establecido producto del temor, la accesibilidad y la ansiedad que genera el perder la presencialidad y confiabilidad en el proceso de enseñanza-aprendizaje.

\section{Referencias}

Balladares-Burgos, J. (2018). Diseño pedagógico de la educación digital para la formación del profesorado. Relatec: Revista Latinoamericana de Tecnología Educativa, 17(1), 41-60. https://doi. org/10.17398/1695-288X.17.1.41

Barrantes, R. (2015). Métodos de estudio a distancia e investigación: a la búsqueda del conocimiento científico. EUNED.

Blancafort, C., González, J., Sisti, O. y Rivera-Vargas, P. (2019). El aprendizaje significativo en la era de las tecnologías digitales. En P. Rivera-Vargas, P. Neut, S. Pascual, P. Lucchini y P. Prunera (Eds.), Pedagogías Emergentes en la Sociedad Digital. Vol. 1 (pp. 49-60). LiberLibro. http://diposit.ub.edu/dspace/bitstream/2445/133194/4/Pedagogi\%CC\%81as\%20emergentes\%20 en\%20la\%20sociedad\%20digital.pdf

Calderón, M., y Loja, H. (2018). Un cambio imprescindible: el rol del docente en el siglo XXI. Illari, 6, 35-38. https://revistas.unae. edu.ec/index.php/illari/article/view/284 
Cardona-Londoño, C., Ramírez-Sánchez, M. y Rivas-Trujillo, E. (2020). Educación superior en un mundo virtual, forzado por la pandemia del Covid 19. Espacios, 41, 44-57. https://www.revistaespacios.com/a20v41n42/20414226.html

Espinoza, E., Ley, N., y Guamán, V. (2019). Papel del tutor en la formación docente. Revista de Ciencias Sociales, 25(3), 230-241. Recuperado de https://www.redalyc.org/articulo.oa?id=28060161020

Espinoza, E., Ley, N., y Guamán, V. (2020). Aprendizaje cooperativo y la Web 2.0. Universidad Técnica de Machala. Revista Ibérica de Sistemas e Tecnologías de Informação, 25, 520-538. https:// www.researchgate.net/publication/338852379_Aprendizaje_ cooperativo_y_la_Web_20_Universidad_Tecnica_de_Machala

Granda, L., Espinoza, E. y Mayon, E. (2019). Las TIC como herramientas didácticas del proceso de enseñanza-aprendizaje. Revista Conrado, 15(66), 104-110. http://conrado.ucf.edu.cu/index. $\mathrm{php} /$ conrado

Hermosa, P. (2015). Influencia de las tecnologías de información y comunicación (TIC) en el proceso enseñanza-aprendizaje: una mejora de las competencias digitales. Revista Cientifica General José María Córdova, 13(16), 121-132. https://doi. org/10.21830/19006586.34

Iglesias, M. J., Lozano, I., y Martínez, M. A. (2013). La utilización de herramientas digitales en el desarrollo del aprendizaje colaborativo: Análisis de una experiencia en educación superior. REDU : Revista de Docencia Universitaria, 11(2), 333-351. https://polipapers.upv.es/index.php/REDU/article/view/5579/5569

López-Neira, L. R. (2017). Indagación en la relación aprendizaje-tecnologías digitales. Educación y Educadores, 20(1), 1-105. http://www.scielo.org.co/pdf/eded/v20n1/0123-1294eded-20-01-00091.pdf

Márquez, E., y Mancilla, F. (2018). La aplicación del aprendizaje meta-cognoscitivo y las tecnologías digitales en la educación universitaria. Administración y Organizaciones, 19(36), 13-34. https://rayo.xoc.uam.mx/index.php/Rayo/article/view/41

Monasterio, D. y Briceño, M. (2020). Educación mediada por las tecnologías: un desafío ante la coyuntura del Covid-19. Observador Del Conocimiento, 5(1), 100-108. http://www.oncti.gob.ve/ojs/ index.php/rev_ODC/article/view/132 
Moreno-Correa, S. (2020). La innovación educativa en los tiempos del Coronavirus. Salutem Scientia Spiritus, 6(1),14-26. https://pesquisa.bvsalud.org/portal/resource/pt/biblio-1087909

Ñáñez-Rodríguez, J., Solano-Guerrero, J., y Bernal-Castillo, E. (2019). Ambientes digitales de aprendizaje en educación a distancia para la formación inicial de docentes: percepciones acerca de su pertinencia. Revista de Investigación, Desarrollo E Innovación, 10(1), 107-119. https://doi.org/10.19053/20278306.v10.n1.2019.10015

Ramírez, U. y Barragán, J. (2018). Autopercepción de estudiantes universitarios sobre el uso de tecnologías digitales para el aprendizaje. Apertura, 10(2), 94-109. https://doi.org/10.32870/ ap.v10n2.1401

Verdecia, E., Enríquez, S. C., Gargiulo, S. B., Ponz, M. J., Scorians, E. E., Vernet, M. y Wenk, N. (2015). Tecnologías de la información y las comunicaciones en educación. Logros actuales y proyección hacia el futuro. Revista Cubana de Educación Superior, 34(2), 4-17. http://scielo.sld.cu/scielo.php?script=sci_arttext\&pid=S0257-43142015000200001\&lng=es\&tlng=es

Villacres, G., Espinoza, E., y Rengifo, G., (2020). Empleo de las Tecnologías de la Información y la Comunicación como estrategia innovadora de enseñanza y aprendizaje. Revista Universidad y Sociedad, 12(5), 136-142. https://rus.ucf.edu.cu/index.php/rus/ article/view/1691/1693 
